\title{
Role of admixtures on dissociation of molecular gases
}

\author{
V. Kudrle, P. Botoš, A. TÁlskÝ \\ Department of Physical Electronics, Masaryk University \\ Kotlářská 2, CZ-61137 Brno, Czech Republic
}

Received 26 April 2004

It is known that dissociation degree in the afterglow can be increased substantially if a small admixture is added to to the main gas prior to its passage through the discharge. In this experimental work we measured the influence of the admixture on dissociation degree, when it is added directly into the afterglow.

$P A C S:$ 52.70.Gw, 82.33.Xj

Key words: afterglow, EPR, dissociation, admixture

\section{Introduction}

Many authors $[1,2,3,4,5]$ demonstrated that even a small admixture can dramatically change the dissociation of the gas. This phenomenon is generally supposed to be caused by one or more of the following effects: change of reduced electric field $E / N$ in the discharge, various homogeneous reactions or by decrease of the wall recombination coefficient due to heterogeneous reactions. All these hypotheses are either directly or indirectly based on the fact, that the admixture must be present in the active discharge.

However, we found [6] that similar effect exists, even with a smaller magnitude, when the admixture is added downstream from the discharge. In this case the assumptions, on which above mentioned hypotheses are based, are not valid any more. To understand better this phenomenon we compared the influence of admixture added to the afterglow with the case when its added upstream from the discharge.

\section{Experimental}

A microwave discharge is produced in a quartz discharge tube with inner diameter of $13 \mathrm{~mm}$ by means of surfatron cavity, powered by $50 \mathrm{~W}$ magnetron working at $2.45 \mathrm{GHz}$. Our experiments being carried out in a flowing regime, afterglow is observed downstream in $1 \mathrm{~m}$ long quartz tube with inner diameter of $8 \mathrm{~mm}$. Small amount of admixture may be added either to a main gas before its passing through the discharge, either through a capillary directly into afterglow.

The gases are led from gas cylinders through the mass flow controllers to a $\mathrm{LN}_{2}$ cold trap, which removes traces of unwanted impurities, like a water or hydrocarbons. The purities of all used gases were better than $99.995 \%$ according to manufacturers' certificates. Flow rate of admixture was varied between 0 and $10 \mathrm{sccm}$ and the flow rate of main gas being kept at $100 \mathrm{sccm}$ for nitrogen and $40 \mathrm{sccm}$ for 
oxygen. The pressure in the discharge was $400 \mathrm{~Pa}$ and $200 \mathrm{~Pa}$, respectively. Total desorption and leak rate was around $5 \times 10^{-3} \mathrm{sccm}$. We therefore estimate the impurity content to be under $100 \mathrm{ppm}$.

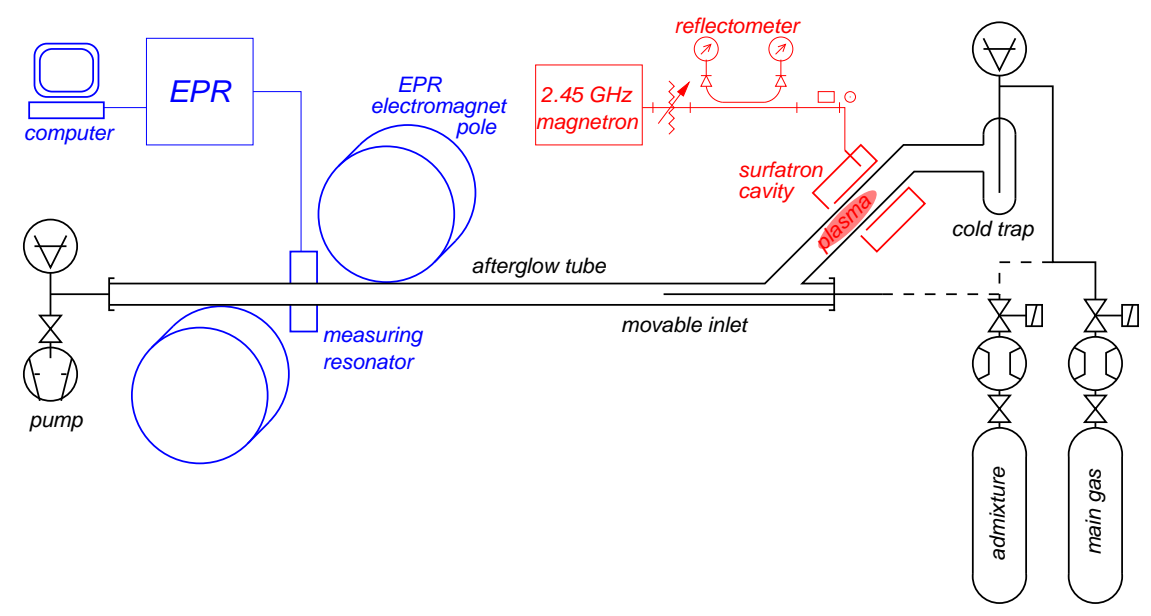

Fig. 1. Schematic drawing of the experimental apparatus.

We employed electron paramagnetic resonance (EPR) spectrometer JEOL JES3B operating in $\mathrm{X}$-band to measure the concentration of atomic species. This method is based on resonance absorption of microwave energy by the transitions between Zeeman split levels. For concentration measurements we used the ground level. In the case of atomic nitrogen, it is ${ }^{4} S_{3 / 2}$ and in the case of oxygen it is ${ }^{3} P_{1,2}$. After a calibration of the EPR device by molecular oxygen [7], which is paramagnetic, the absolute concentration was obtained. The advantage of such calibration is well defined fill factor, which is not easy to achieve with traditional DPPH standard. In comparison with other techniques it has a big advantage in its non-invasivity and the ability to determine the absolute concentrations of wide range of species [8].

\section{Results and discussion}

Addition of small amount of oxygen impurity into nitrogen gas prior to its passage through the discharge may give raise to more than ten-fold increase in $\mathrm{N}$ atom concentration in the afterglow, as demonstrated in Fig. 2. This effect was observed also for other main gases and admixtures $\left(\mathrm{N}_{2}, \mathrm{O}_{2}, \mathrm{H}_{2}\right)$.

Often, such increase of dissociation degree for small admixture percentage is explained by a change of reduced electric field $E / N[9]$, various homogeneous reactions [10] or by a decrease in the wall recombination coefficient [11].

These hypotheses were developed for admixture going through the active discharge but it was found [6] that certain increase in $[\mathrm{N}]$ is observable even when admixture is added directly into the afterglow. In such case the assumptions on which these theories are based may not be valid. 
Role of admixtures on dissociation of molecular gases

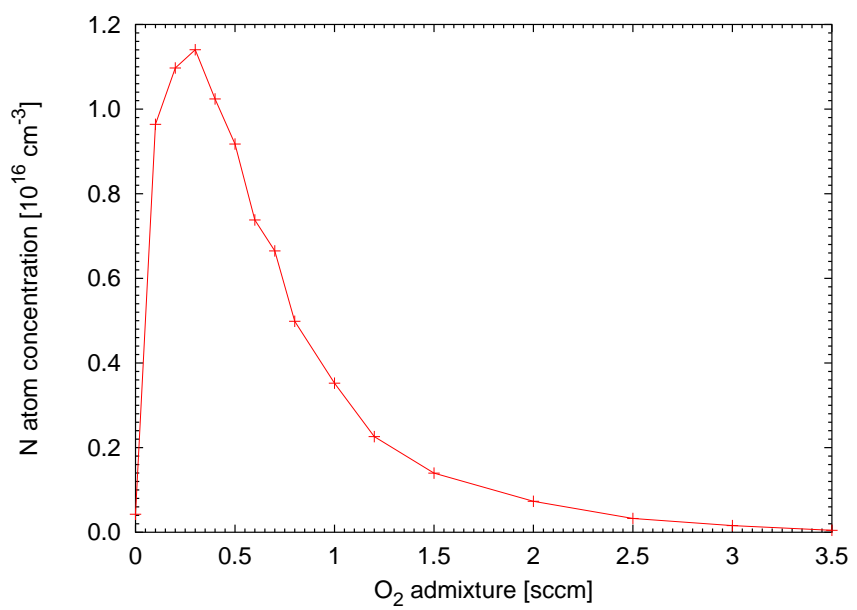

Fig. 2. Influence of oxygen admixture added upstream (before the discharge) to nitrogen gas on $\mathrm{N}$ atom concentration in afterglow.

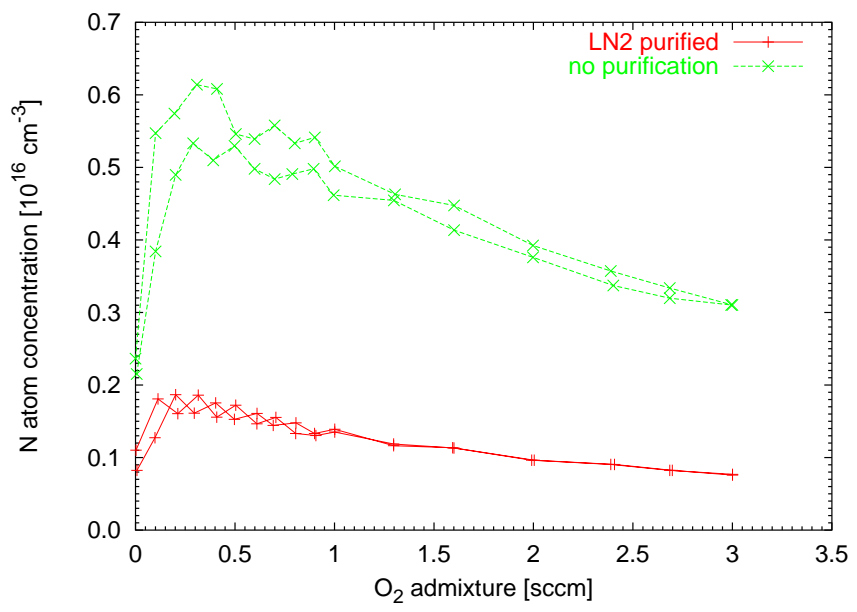

Fig. 3. Influence of oxygen added downstream (into the afterglow) to nitrogen gas on $\mathrm{N}$ atom concentration in afterglow. One curve corresponds to the case when the nitrogen gas passed through $\mathrm{LN}_{2}$ trap, the second one to the case when it did not.

Experimental results of adding the oxygen into nitrogen afterglow are presented in Fig. 3. We observe the steep rise of atomic nitrogen density with for low oxygen admixtures as in the case of upstream mixing, albeit smaller. Despite the fact, that total impurity content is below $100 \mathrm{ppm}$, there is pronounced effect [12] on nitrogen dissociation. It is demonstrated by the changes of nitrogen atom density when the nitrogen gas is additionally purified by passing through $\mathrm{LN}_{2}$ cold trap. It is interesting to note that oxygen admixture influence does not 'add' with impurities 
influence, but is somewhat "multiplied" by their presence.

We performed the same experiment also with gases inverted, i.e. with oxygen main gas and with nitrogen as the admixture. The results for introducing the nitrogen upstream from the discharge are presented in Fig. 4. The behaviour is nearly the same as for the Fig. 2, only the decrease of oxygen atom density for higher admixture flows is rather small.

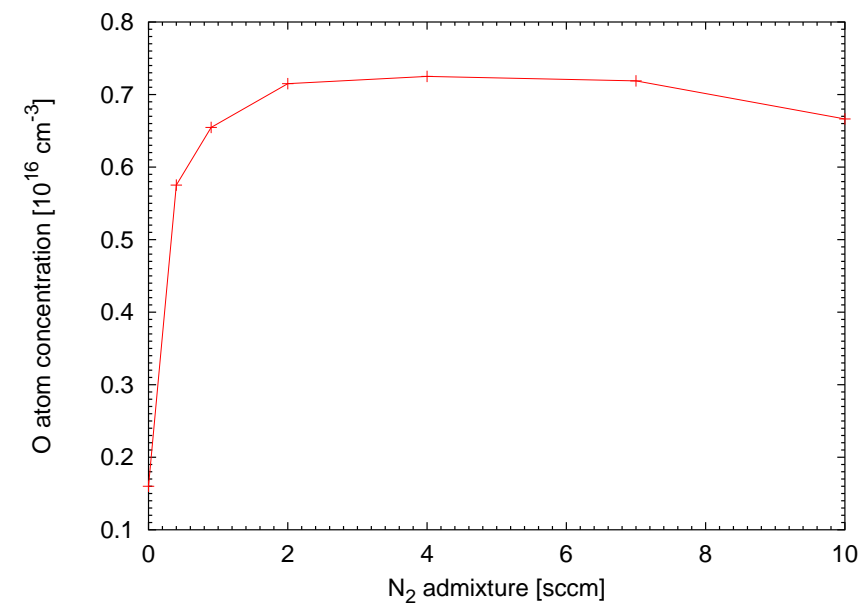

Fig. 4. Influence of nitrogen admixture added upstream (before the discharge) to oxygen gas on $\mathrm{O}$ atom concentration in afterglow.

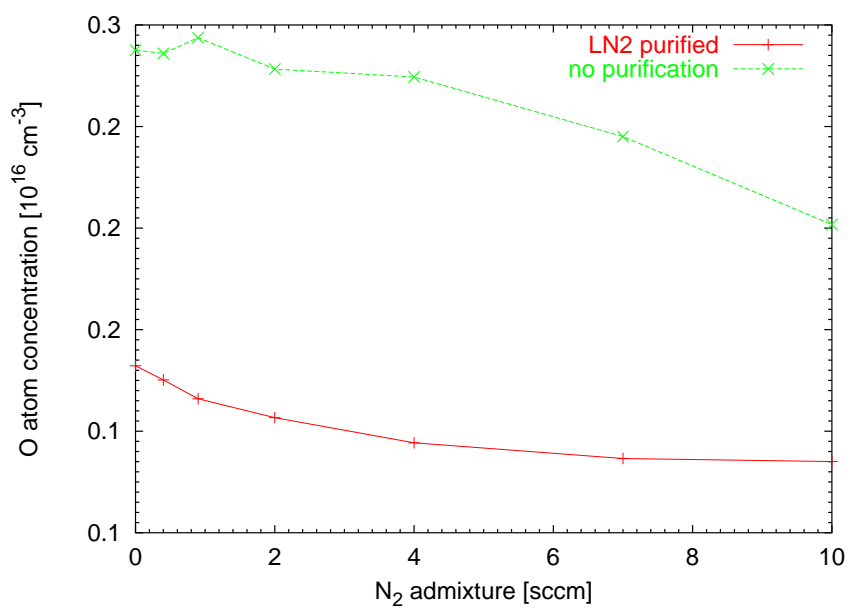

Fig. 5. Influence of nitrogen added downstream (into the afterglow) to oxygen gas on $\mathrm{O}$ atom concentration in afterglow. One curve corresponds to the case when the oxygen gas passed through $\mathrm{LN}_{2}$ trap, the second one to the case when it did not. 
However, Fig. 5 shows that atomic oxygen concentration is nearly independent of nitrogen admixture.

The theories, mentioned above, which explain the rise of dissociation degree by admixtures present in the active discharge are not applicable to the case of admixture injecting into the afterglow. There are two possibilities: either are new atoms produced or the atoms already produced in the discharge cannot recombine back to molecules.

The atom producing volume reaction needs energy. The only energy available in sufficient quantity is in the form of metastable molecules.

Heterogeneous process responsible for decreasing recombination probability is probably the occupation of active sites [13] by admixture particles. As recombination occurs preferably at these active sites, their occupation leads to decreased wall recombination. In such way the observed curves were explained in [14].

However, from the results of [4] it seems that the recombination coefficient of the nitrogen atoms is so small, that even if it is set to zero by heterogeneous reaction, the resulting increase in nitrogen atom density is negligible. Therefore in the case of nitrogen the cause of dissociation degree should be attributed to the presence of metastable molecules. However, why the same effect does not take place in the case of oxygen, remains a question.

\section{Conclusions}

Impurities added in relatively small quantities to a molecular gas upstream from the discharge enhance the dissociation degree in the afterglow. In nitrogen, such effect is observed also when the admixtures are added into the afterglow, but smaller magnitude. It is mainly caused by a collisional transfer of energy from metastables to dissociation. In oxygen the addition of admixtures to afterglow do not play any role.

This work was supported by Czech Ministry of Education under contract number MSMT 143100003.

\section{References}

[1] R. A. Young, R. L. Sharpless, R. Stringham: J. Chem. Phys. 40 (1964) 117.

[2] A. R. De Souza, C. M. Mahlmann, J. L. Muzarl, C. V. Speller: J. Phys. D: Appl. Phys. 26 (1993) 2164.

[3] A. Granier, D. Chereau, K. Henda, R. Safari, P. Leprince: J. Appl. Phys. 75 (1) (1994) 104.

[4] V. Zvoníček: Study of the surface recombination of oxygen, nitrogen and hydrogen atoms Thesis, Masaryk University, Brno, 1997.

[5] G. Cartry: Etude par spectroscopie de la cinetique des decharges electriques dans les melanges $\mathrm{N}_{2}-\mathrm{O}_{2}$, Thesis, Universite Paris Sud, Orsay, 1999. 
[6] V. Kudrle, P. Vašina, A. Tálský, J. Janča: Czech. J. Phys. 52-D (2002) 589.

[7] S. Krongelb, H. W. P. Strandberg: J. Chem. Phys. 31 (1959) 1196.

[8] A. A. Westenberg: Prog. React. Kinet. 7 (1973) 23.

[9] J. Nahorny: Etude de la cinetique d'une decharge electrique dans les melanges Azote - Oxygene Thesis, Université Paris Sud, Orsay, 1994.

[10] J. Nahorny, C. M. Ferreira, B. Gordiets, D. Pagnon, M. Touzeau, M. Vialle: J. Phys. D: Appl. Phys 28 (1995) 738.

[11] B. Gordiets, C. M. Ferreira, J. Nahorny, D. Pagnon, M. Touzeau, M. Vialle: J. Phys. D: Appl. Phys. 29 (1996) 1021.

[12] K. M. Evenson, D. S. Burch: J. Chem. Phys. 45 (1966) 2450.

[13] A. Zangwill: Physics at Surfaces, Cambridge Univ. Press, Cambridge UK, 1988.

[14] V. Kudrle, A. Tálský, A. Kudláč, V. Křápek, J. Janča: Czech. J. Phys. 50-S3 (2000) 305. 